\title{
The impact on health status in short- and long-terms of a novel and non-orthodox real-world COPD rehabilitation effort in rural India: an appraisal
}

This article was published in the following Dove Press journal: International Journal of COPD

Parthasarathi Bhattacharyya'

Rupak Ghosh'

Dipanjan Saha'

Bodhisattwa Chakraborty'

Pallav Bhattacharyya'

Madan Sarma'

Saibal Mazumdar ${ }^{2}$

Kajal Chatterjee ${ }^{2}$

Avijit Chowdhury ${ }^{3}$

'Department of Pulmonology, Institute of Pulmocare and Research, Kolkata, India; ${ }^{2}$ Department of General Medicine, Liver Foundation, Kolkata, India; ${ }^{3}$ Gastroenterology Department, Liver Foundation, Kolkata, India
Correspondence: Parthasarathi Bhattacharyya

Department of Pulmonology, Institute of Pulmocare and Research, DG-8, Action Area I, New Town, Kolkata, West Bengal, 700I56, India

Tel +9l 8017990424

Email parthachest@yahoo.com
Background: Rehabilitation has been an integral part of management of COPD. Since the implementation of the standard rehabilitation protocol is hardly possible in the rural developing world, aiming to make a feasible alternate effort may be worthwhile.

Methods: COPD patients diagnosed through spirometry were first stabilized with 6 weeks of uniform pharmacotherapy. Subsequently, they were subjected to a curriculum-based intensive single-session intervention with education, bronchial hygiene, and exercise training. The latter involved whole body exercise, pursed lip breathing, and diaphragmatic exercise. The participants continued to practice the exercises under real-world encouragement and supervision from trained volunteers. The impact was appraised in terms of change in health status through COPD assessment test (CAT) score measurements at stabilization, and after 6 weeks and 1 year of the intensive training and education.

Results: At stabilization, 70 out of 96 selected COPD subjects (73\%) turned up (with mean age $62 \pm 9$ years and mean $\mathrm{FEV}_{1}$ as $1.16 \pm 0.39 \mathrm{~L}$ ) showing improvement as per CAT score ( $p=0.0001$ ) from pharmacotherapy. After practicing the imparted education and training for 6 weeks, all these 70 participants had further significant improvement in the health status ( $\mathrm{n}=70, p=0.00001$ ). This improvement, been reinforced and supervised, continued to last even at 1 year $(\mathrm{n}=54, p=0.0001)$.

Conclusion: The self-managed practice of a single-session education and training under real-world supervision can bring forth significant long-term improvement in the health status of COPD sufferers. Such simple and feasible intervention may substitute formal COPD rehabilitation programs in resource constraint situations.

Keywords: rural COPD, extensive training, single point intensive education and training, quality of life, respiratory function tests

\section{Introduction}

Despite the increasing prevalence of COPD globally, ${ }^{1}$ and its presence being significant in India, ${ }^{2-5}$ the information regarding the disease and its treatment status especially from rural areas appears scanty. ${ }^{6}$ There are as yet no published data on the nonpharmacological therapy of this debilitating disease from the rural developing world. Here, we present our effort to appreciate the effects of a novel, self-managed, and supervised real-world practice of an intensive single-session education and training based on a simple curriculum for the COPD patients in rural India. 


\section{Methods}

The study proposal was first ethically approved by the institutional ethics committee, "Institutional Ethics Committee of the Institute of Pulmocare and Research". The study was accomplished in several steps as depicted in the following text (Table 1).

\section{Deciding the modus operandi, selection, and training of volunteers}

We had experience of holding several COPD educations and training camps in our center and in the same district before. The experience helped us to plan the details of operations objectively with the help of another philanthropic organization (West Bengal Liver Foundation) being engaged in the same geographic area. Through critical analysis of our strengths and the possible hindrances with their way outs in several meetings, we drafted the blueprint of the operation. We selected the curriculum been prepared by us in Bengali (the local language) earlier through several field trials with the support of the Chest Foundation Award 2009. The curriculum consisted of basics of COPD with role of smoking cessation, information regarding the proper medications, the correct use of the inhalational medicines, and importance of treatment of associated ailments in a simple language. It also contained the proper and feasible nutritional and hygienic interventions (including the bronchial hygiene), exercise in general with special emphasis on walking and breathing exercises (pursed lip breathing and diaphragmatic breathing) with necessary pictorial display.

We focused on the feasibility issues at the very planning stage and thus selected seven volunteers belonging to seven villages of the Rajnagar Block of the Birbhum district. They all happened to be rural health care providers keen to learn and join our effort. We planned to utilize the relationship and influence of them on the local population to implement our plan successfully. They were subsequently educated about COPD with its basic qualities and non-pharmacological treatment. We discussed with them about the protocol, the curriculum of education and training, and their role in the whole research including transferring data electronically and supervising the participants following our interventions. Thereafter, they were brought to the state capital and were given formal training by the Indian Chest Society with certification as spirometry technician. Their proficiency was subsequently ensured through repeated practice and performance at our Kolkata center and also at the field in their villages.

The actual performances of the project was done through the following steps that were initiated from July, 2012 to February, 2014.

\section{The detection of COPD patients and inclusion for intervention}

This was accomplished by performing spirometry following the American Thoracic Society guideline in people older than 40 years and having any of the respiratory symptoms as shortness of breath, cough, expectoration, wheeze, etc., in a door-to-door survey. The validity and the reliability of all the spirometry tracings were checked by the consultants at the institute using internet. The severity of airflow limitations were noted following the GOLD guideline. ${ }^{1}$

The willing patients joined the study by signing written informed consent form. Patients older than 75 years, having a history of exacerbation in the preceding 6 weeks, or reluctance to adhere to the protocol that demands repeated visits at camp sites, or having any significant physical limitations (eyesight, speech, hearing, mobility) and comorbidities (as left ventricular failure, acute cor pulmonale, overt liver or

Table I Action plan for accomplishment of the job in steps

\begin{tabular}{|c|c|c|}
\hline Steps & \multicolumn{2}{|l|}{ The action plan } \\
\hline I & \multicolumn{2}{|c|}{ Deciding the modus operandi, selection, and training of volunteers } \\
\hline & \multicolumn{2}{|r|}{$\downarrow$} \\
\hline 2 & \multicolumn{2}{|c|}{ The detection of COPD sufferers and inclusion for intervention } \\
\hline & \multicolumn{2}{|r|}{$\downarrow$} \\
\hline \multirow[t]{2}{*}{3} & The first camp visit & General appraisal with medication alone intervention \\
\hline & \multicolumn{2}{|r|}{$\downarrow 6$ weeks } \\
\hline \multirow[t]{2}{*}{4} & The second camp visit & Appraisal of the impact of pharmacotherapy and "education and training" intervention \\
\hline & \multicolumn{2}{|r|}{$\downarrow 6$ weeks } \\
\hline \multirow[t]{2}{*}{5} & The third camp visit & $\begin{array}{l}\text { Appraisal of the impact of practicing the "education and training" intervention and } \\
\text { reiterating the same }\end{array}$ \\
\hline & \multicolumn{2}{|r|}{$\downarrow 6$ weeks } \\
\hline 6 & The fourth camp visit & $\begin{array}{l}\text { Reappraisal of the impact of practicing the "education and training" intervention and } \\
\text { reiterating the same }\end{array}$ \\
\hline
\end{tabular}


renal failure, disseminated malignancy, psychiatric illness, etc.) as apparent from the history, clinical examination, and investigations (whenever available) were excluded. A chest $\mathrm{X}$-ray and a 12-lead ECG were done for each patient before final inclusion. It was the physician's prerogative to exclude any patient based on clinicoradiological or electrocardiographic pictures at the first visit by the patient.

\section{The actual performance of the interventions}

The actual performance was distributed in a series of camps at every 6 weeks. The dates were fixed on Sundays for convenience. At each such preselected date, the participants were brought to a selected venue (camp) (Aligarh Sesalfirm School, Rajnagar, Birbhum, West Bengal) with the help of the volunteers. The activities done subsequently are listed below.

\section{The camp visits}

This first camp-site activities (visit for evaluation in medication alone intervention) included a series of jobs done in a systematic way at multiple tables arranged in such a fashion as to allow the patients move in a pre-determined direction. This helped to accomplish 1) registration; 2) measurement of blood pressure, body mass index (BMI), resting pulse rate, and arterial oxygen saturation; 3) assessment of the health status with COPD assessment test (CAT); 4) actual examination by an expert pulmonologists; 5) distribution of medicines with demonstration of the technique of using the dry powder inhalers followed by one-to-one training to use the device properly; and 6) offering refreshment and arrangement for a follow-up after 6 weeks. This visit was named "the medication alone intervention visit".

The prescriptions included combination of dry powder inhalers as salbutamol + ipratropium (Salbair-I; Lupin Limited, Mumbai, India) and budesonide + formoterol (Budamate; Lupin Limited) along with the inhalation device (Lupihaler; Lupin Limited) with the treatment of comorbidities, if any.

Reviewing the patients at the second visit ("medication plus education and training intervention visit") was done after a gap of 6 weeks. It included re-evaluation in the same fashion as in the first visit with documentation of the CAT score again. This time, in addition to offering advice, the patients were further imparted with intensive education and training using the simple and feasible syllabus mentioned.

The process included a common interactive session with an experienced pulmonologist having good communicating skill. The patients were educated about the role of walking as a general whole body exercise and were advised for regular walking (with hands free) routinely at least for two session a day (morning and evening) till they feel tired or near-exhausted. They were also taught the technique of using inhalers, breathing exercises especially the pursed lip breathing and diaphragm exercises along with the role of bronchial hygiene. These exercises were demonstrated and thereafter a few patients were randomly selected from the crowd to perform one or the other job and demonstrate it to the rest. Thus, the methods were repeated and rehearsed. Whenever possible, a practical action (viz. correct inhalation that leads to instant relief of symptoms) was demonstrated in some of the patients in order to convince the others and ensure compliance from all. Demonstrating the paradoxical breathing in a patient and explaining the difference from the normal breathing with simultaneous participation of one of our healthy volunteers was done. Following that, the patients were addressed in one-to-one interaction session in multiple groups each having a resource person.

Thereafter, they were provided with therapy till the next visit after 6 weeks with request to follow-up and seek assistance from the volunteers for any enquiry or support in between. Each volunteer (rural health care provider) was also entrusted openly in front of all to supervise the patients of his village regularly.

Reviewing them at the third visit ("appraisal and re-training intervention visit") was done after a gap of another 6 weeks and it included the recording of the CAT score and reiteration of the same education and training been imparted previously.

Reviewing them again after 1 year ("re-appraisal and re-training intervention visit") was done with the same group of patients who remained under the supervision of the rural volunteers without any fixed protocol. We recorded the CAT score again and reiterated the same education and training once again.

Each and every member of the team was consistently delegated with a particular job (like registration, examinations, assisting the documentation of the CAT scoring, etc.) in order to maintain uniformity at each step in all the visits.

Data compilation and analysis were done after four successive visits were held and the statistical analysis was done using paired Student's $t$-tests.

\section{Results}

Out of 143 subjects been diagnosed as COPD at their homes, 47 COPD sufferers were excluded (see Figure 1 for the causes). The mean \pm SD age of the 96 subjects who joined the study was $62 \pm 9$ years, the majority were male $(n=68)$, 


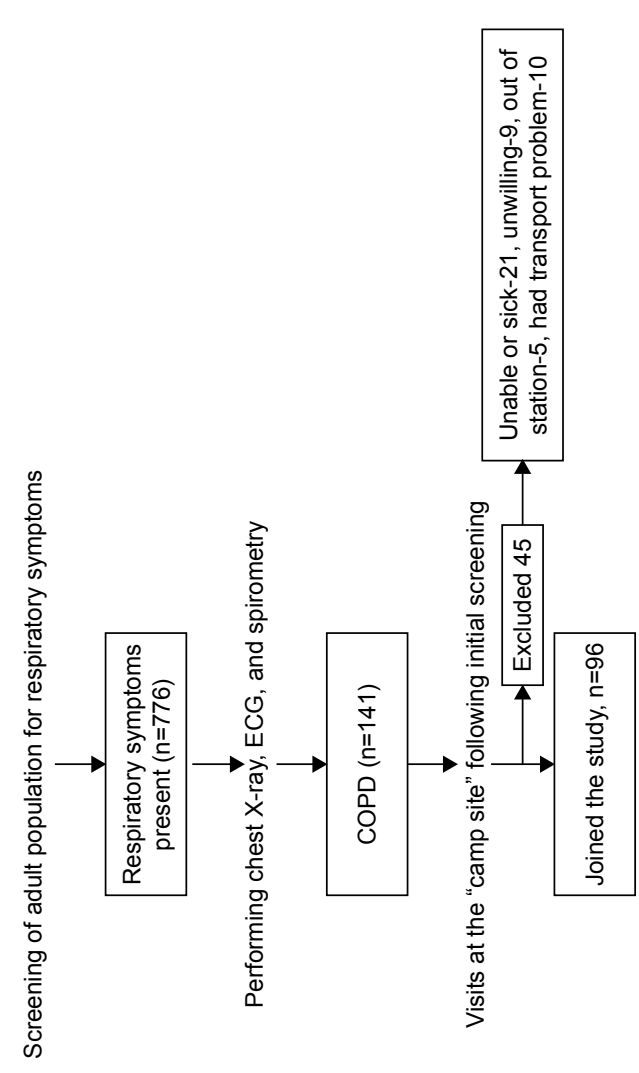

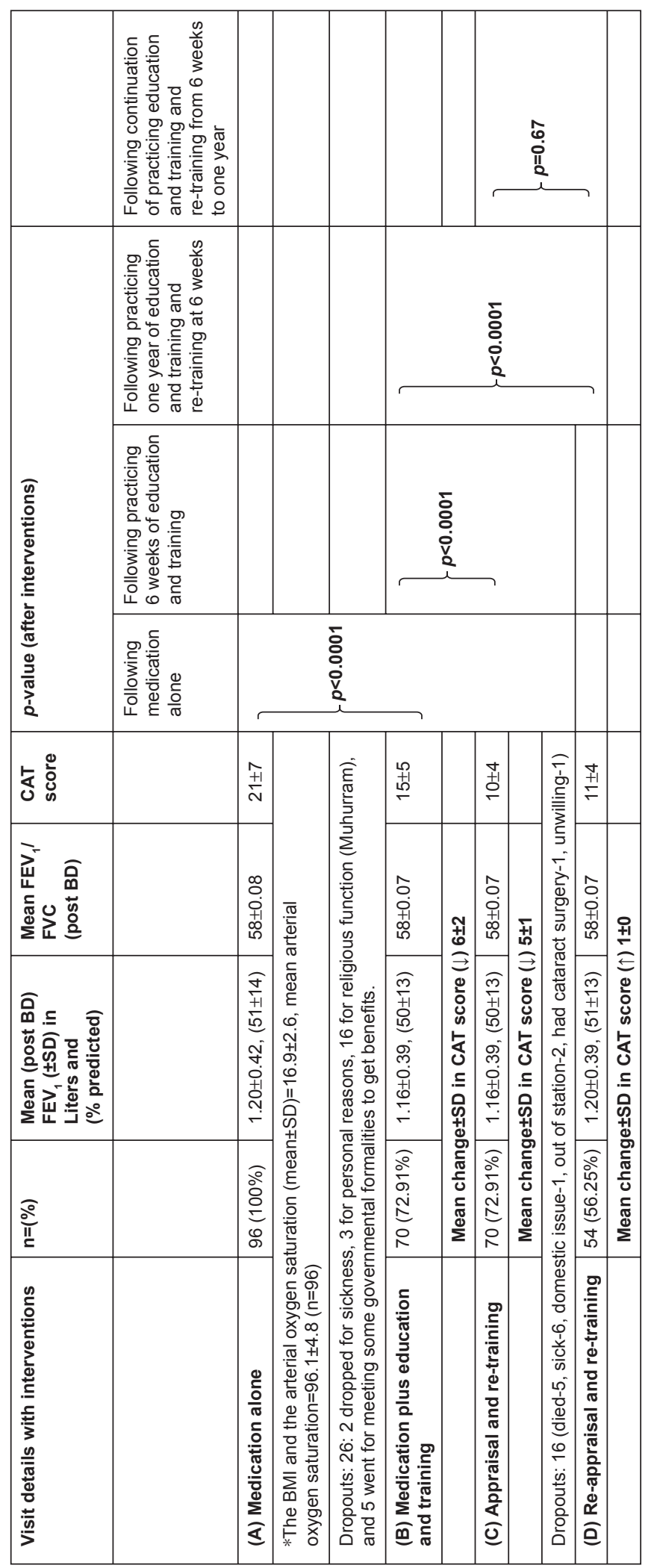

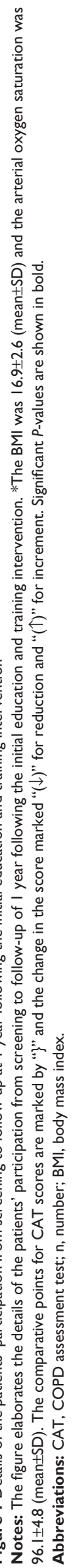


and the mean $\pm \mathrm{SD}$ post bronchodilator $\mathrm{FEV}_{1} / \mathrm{FVC}$ and $\mathrm{FEV}_{1}$ values of the participants were $58.9 \pm 8$ and $1.20 \pm 0.42 \mathrm{~L}$, respectively. All of them attended the "medication alone intervention". Out of them, 70 patients (mean BMI $16.9 \pm 2.6 \mathrm{~kg} / \mathrm{m}^{2}$, mean arterial oxygen saturation $96.1 \pm 4.8 \%$ at rest; the reason of the dropouts is elaborated in Figure 1) attended the successive camps (the "medication plus education and training intervention visit" and the "appraisal and retraining intervention visit") after every 6 weeks (Figure 1). Again after 1 year, 54 out of these 70 patients (Figure 1 with causes of dropouts) attended the "re-appraisal and retraining the interventions". The impact of the interventions was calculated in terms of CAT score at each step as been displayed in Figure 1. The first or the "medication alone intervention" resulted in a statistically significant improvement (CAT score falling from $21 \pm 7$ to $15 \pm 5$ over 6 weeks; $p=0.00001)$. The health status further improved significantly $(\mathrm{n}=70, p=0.0001)$ following the intervention (medication with education and training) being measured at the third camp visit (see Figure 1) and this improvement continued to exist even at 1 year $(n=54$, $p=0.0001$ ) at the fourth camp visit (Figure 1).

\section{Discussion}

We have observed an impressive improvement $(p<0.0001)$ (Figure 1) in the health status following the interventions (Figure 1). The significant drop in CAT score $(p<0.0001)$ following the first "medication alone" intervention suggests that the participants had been treated very poorly as per the pharmacotherapy of COPD was concerned prior to our intervention. The CAT score fell further $(p<0.0001)$ after 6 weeks of the single session of "medication plus education and training" intervention. Since the patients had been on the same pharmacotherapy, the impact of the intervention was clearly discernable. The positive effect of the intervention continued to exist even after 1 year $(p<0.0001)$ (Figure 1$)$ in those who attended $(n=54)$ our re-appraisal camp. They continued to practice the education and training imparted at the second visit, which was further reinforced at the subsequent visits. The response from the recipients appeared overwhelming. The dropout of $27 \%$ at the second visit (from 96 to 70 ) was largely (over $80 \%$ ) because of unavoidable factors that were not perceived at planning (religious event and government program [Table 1]). The results, thus, clearly indicate the feasibility and success of the rehabilitation efforts.

COPD rehabilitation, a well-established and useful intervention, is largely not possible in rural India for logistic reasons, while the burden of COPD in the country has been huge ${ }^{2-4}$ causing significant mortality and disability. ${ }^{7,8}$ In addition to the strong evidence favoring the conventional efforts $^{9-13}$ it has been found that any intervention for COPD rehabilitation (both outdoor and home based) appears useful in short of an ideal program. ${ }^{12,14-19}$ Hence, we decided to try for the best feasible rehabilitation of these rural patients despite the adverse ground realities.

The benefits of a outpatient rehabilitation program can persist up to 2 years in terms of reduction in exacerbations, improvement in perception of dyspnea, and health-related quality of life (HRQOL). ${ }^{12}$ Again, outpatient-based COPD education, breathing retraining, and exercise training have shown to improve significantly the self efficacy, exercise endurance, and quality of life score. ${ }^{14}$ A home-based program can improve the exercise endurance and quality of life in long-term (18 months) with reduction in dyspnea. ${ }^{15}$ Simple home-based exercise training program using the shuttle walking test is also found to improve basal dyspnea and quality of life. ${ }^{16}$ Rehabilitation effort using minimal resources has been found equivalent even to the outcome of a center-based program. ${ }^{17}$ Two short-term (6 and 4 weeks, respectively) home-based rehabilitation efforts from India showed beneficial effects on quality of life and exercise endurance. $^{18,19}$

Considering the logistic reality, we decided to try a single-session, curriculum-based, structured, and organized intervention with COPD-related education and training for rehabilitation. The whole process was essentially been a selfmanaged and locally supervised real-world care in rural area of the developing world. For simplicity of performance, we incorporated $\mathrm{CAT},{ }^{20}$ a valid tool for the assessment of the COPD-related health status with efficacy being comparable to St George's Respiratory Questionnaire. ${ }^{21}$ This easy-touse test appears to behave uniformly across countries. ${ }^{22}$ Our resource people were thoroughly experienced and comfortable to assist the collection of response from patients on CAT score.

COPD rehabilitation in rural areas is not new and people, so far, have tried to replicate the formal evidence-based COPD rehabilitation program in rural areas. ${ }^{23}$ Effort intensive program for implementation at the primary care level following evidence-based guidelines resulted in a significant positive impact in several parameters such as HRQOL, health status, changes in practice behavior, improvement in smoking cessation, and health care utilization. ${ }^{24}$

Despite the encouraging results, we observe certain weaknesses in our study. A randomization at the second visit to make a parallel assessment with or without the add-on intervention of education and training was rejected at the planning stage and since that could lead to a notion of discrimination amongst the participants. We decided to step-up of the interventions 
serially (initially pharmacotherapy alone and then with add-on practice of education and training to pharmacotherapy). This allowed the same cohort of patients to act as historical controls for themselves. We could not include an exercise test mainly for safety concern since the arrangement of resuscitative items including emergency oxygen supplementation deemed difficult in those remote villages. We have not taken into account the variables that can affect the care and well-being of our patients; a systematic analysis of the impact of several personal, socioeconomic, and other factors remains issues for future research to provide an insight regarding their compounding effects on the disease and the interventions. Moreover, we have not analyzed the effects of such intervention on smoking cessation and have not considered the end points such as changes in shortness of breath (dyspnea scale), functional capacity (6-minute walk test), frequency of exacerbations, health care utilization, etc. Those could have been worthwhile adjuncts to appreciate the effect of the interventions mentioned. To us, they were not possible within our capacity and resources in the concerned ground reality. The strategy of involving local rural health care providers as our volunteers had turned out as a worthwhile decision. Possibly, the supervision part was very well taken care of by them.

The implication of the observation is far reaching. The scenario of the overall COPD care in rural areas appears to be burdened with poverty, overpopulation, geographical, and sociopolitical adversities often with an overwhelming lack of education and awareness with paucity of adequate health care infrastructure. This ground reality may be the reason of non-availability of any published data on COPD rehabilitation from rural India. The implementation of the conventional structured and evidence-based COPD rehabilitation programs in rural India, thus, appears extremely difficult or practically impossible. Hence, in short of the "gold standard COPD rehabilitation", this simple and feasible effort to support rehabilitation for rural population may turn out to be a useful non-pharmacological adjunct in the management of COPD. We could meet with the challenge of establishing the feasibility of such an effort. Further studies are warranted to validate our effort and help in formulating ways for customization of such innovative non-orthodox programs to apply across varied geo-socioeconomic and cultural realities. The replication of such feasible and effective intervention can help to improve the health status of millions of COPD sufferers.

\section{Conclusion}

The study establishes the feasibility and the success of a simple alternative community-based COPD rehabilitation effort in rural developing world. It proves that even an organized single-session education training can help the COPD patients enormously if it is practiced under supervision of trained volunteers.

\section{Acknowledgments}

We would like to thank the following people for their support in the study: Rana Dey, Institute of Pulmocare \& Research, New Town Kolkata, India; Ratna Dey, Institute of Pulmocare \& Research, New Town Kolkata, India; Jayanta Das, Rural volunteer, Rajnagar, Birbhum, India; Moloy Bhattacharya, Rural volunteer, Rajnagar, Birbhum, India; Pintu Bagdi, Rural volunteer, Rajnagar, Birbhum, India; Kumar Chakarborty, Consultant Physician, Kolkata, India; Ratan Lal Manna, Rural volunteer, Birbhum, India; MD. Nizamuddin, Rural volunteer, Birbhum, India; Dharam Sutradhar, Rural volunteer, Birbhum, India; Sanjoy Mondal, Rural volunteer, Birbhum, India; and Akal Mal, Rural volunteer, Birbhum, India. The Department of Science and Technology of the Government of West Bengal funded the study.

\section{Author contributions}

All authors contributed toward data analysis, drafting and critically revising the paper, gave final approval of the version to be published, and agree to be accountable for all aspects of the work.

\section{Disclosure}

The authors report no other conflicts of interest in this work.

\section{References}

1. Rabe KF, Hurd S, Anzueto A, et al. Global strategy for the diagnosis, management, and prevention of chronic obstructive pulmonary disease: GOLD executive summary. Am J Respir Crit Care Med. 2007;176(6): 532-555.

2. Jindal SK, Aggarwal AN, Gupta D, et al. A review of population studies from India to estimate national burden of chronic obstructive pulmonary disease and its association with smoking. Indian J Chest Dis Allied Sci. 2001;43(3):139-147.

3. Jindal SK, Aggarwal AN, Chaudhry K, et al; Asthma Epidemiology Study Group. A multicentric study on epidemiology of chronic obstructive pulmonary disease and its relationship with tobacco smoking and environmental tobacco smoke exposure. Indian J Chest Dis Allied Sci. 2006;48(1):23-29.

4. Jindal SK. Emergence of chronic obstructive pulmonary disease as an epidemic in India. Indian J Med Res. 2006;124(6):619-630.

5. McKay AJ, Mahesh PA, Fordham JZ, et al. Prevalence of COPD in India: a systematic review. Prim Care Respir J. 2012;21(3):313-321.

6. Mahesh PA, Jayaraj BS, Prahlad ST, et al. Validation of structures questionnaire for COPD and prevalence of COPD in rural areas of Mysore: a pilot study. Lung India. 2009;26(3):63-69.

7. Murray CJ, Lopez AD. Alternative projections of mortality and disability by cause 1990-2020: Global Burden of Disease Study. Lancet. 1997;349(9064):1498-1504.

8. Thornton Snider J, Romley JA, Wong KS, et al. The disability burden of COPD. COPD. 2012;9(5):513-521.

9. Lacasse Y, Goldstein R, Lasserson TJ, Martin S. Pulmonary rehabilitation for chronic obstructive pulmonary disease. Cochrane Database Syst Rev. 2006;4(2):CD003793. 
10. Ries AL, Bauldoff GS, Carlin BW, et al. Pulmonary rehabilitation: joint ACCP/AACVPR evidence-based clinical practice guidelines. Chest. 2007;131(Suppl 5):4S-42S.

11. Goldstein RS, Gork EH, Stubbing D, Avendano MA, Guyatt GH. Randomized controlled trial of respiratory rehabilitation. Lancet. 1994;344(8934):1394-1397.

12. Güell R, Casan P, Belda J, et al. Long-term effects of outpatient rehabilitation of COPD: a randomized trial. Chest. 2000;117(4):976-983.

13. Lacasse Y, Wong E, Guyatt GH, King D, Cook DJ, Goldstein RS. Meta-analysis of respiratory rehabilitation in chronic obstructive pulmonary disease. Lancet. 1996;348(9035):1115-1119.

14. Jung JH, Kim JY. The effects of self-efficacy promoting pulmonary rehabilitation program in out-patients with chronic obstructive pulmonary disease. Tuberc Respir Dis. 2006;61(6):533-546.

15. Strijbos JH, Postma DS, van Altena R, Gimeno F, Koëter GH. Hospitalbased pulmonary rehabilitation program and a home-care pulmonary rehabilitation program in patients with COPD: a follow-up of 18 months. Chest. 1996;109(2):366-372.

16. Hernández MT, Rubio TM, Ruiz FO, Riera HS, Gil RS, Gómez JC. Results of a home-based training program for patients with COPD. Chest. 2000;118(1):106-114.

17. Holland AF, Mahal A, Hill CJ, et al. Home-based rehabilitation for COPD using minimal resources: a randomised, controlled equivalence trial. Thorax. 2016;72(1):57-65.
18. Pande A, Singhal P, Kumar R, Gaur SN. Effect of home-based pulmonary rehabilitation programme on disability in patients with chronic obstructive pulmonary disease. Indian J Chest Dis Allied Sci. 2005; 47(3):217-219.

19. Singh V, Khandelwal DC, Khandelwal R, Abusaria S. Pulmonary rehabilitation in patients with chronic obstructive pulmonary disease. Indian J Chest Dis Allied Sci. 2003;45(1):13-17.

20. Jones PW, Harding G, Berry P, Wiklund I, Chen WH, Kline Leidy N. Development and first validation of the COPD assessment test. Eur Respir J. 2009;34(3):648-654.

21. Jones PW, Quirk FH, Baveystock CM, Littlejohns P. A self-complete measure of health status for chronic airflow limitation. The St. George's Respiratory Questionnaire. Am Rev Respir Dis. 1992;145(6): 1321-1327.

22. Jones PW, Brusselle G, Dal Negro RW, et al. Properties of the COPD assessment test in a cross-sectional European study. Eur Respir J. 2011;38(1):29-35

23. Deprez RD, Kinner A, Baggott LA, Milard P, Moody B. Rural COPD quality improvement initiative. Abstract presented at: APHA 135th Annual Meeting and Expo; November 3-7, 2007; Washington, DC. Abstract \#158397.

24. Rasekaba TM, Williams E, Hsu-Hage B. Can a chronic disease management pulmonary rehabilitation program for COPD reduce acute rural hospital utilization? Chron Respir Dis. 2009;6(3):157-163.
International Journal of COPD

\section{Publish your work in this journal}

The International Journal of COPD is an international, peer-reviewed journal of therapeutics and pharmacology focusing on concise rapid reporting of clinical studies and reviews in COPD. Special focus is given to the pathophysiological processes underlying the disease, intervention programs, patient focused education, and self management protocols.

\section{Dovepress}

This journal is indexed on PubMed Central, MedLine and CAS. The manuscript management system is completely online and includes a very quick and fair peer-review system, which is all easy to use. Visit http://www.dovepress.com/testimonials.php to read real quotes from published authors. 